\title{
Ubiquitous Lighting: The Third Color Digital Art $\times$ Lighting Symposium and Workshop
}

\author{
Kyoko Hidaka \\ Shibaura Institute of Technology \\ Tokyo, Japan \\ khidaka@shibaura-it.ac.jp
}

\begin{abstract}
This paper presents an empirical case study of how a digital art tele-symposium and a student workshop can be held remotely. From December 2020 to March 2021, Shibaura Institute of Technology: Color Communication Design Laboratory in Japan, and Assumption University in Thailand co-hosted a tele-symposium and workshops. This analysis deals with how to integrate art and technology in a remote workshop. The overall theme of the workshop is 'Ubiquitous Lighting'. The works produced during this workshop will be exhibited at the Bangkok Design Week 2021 in May. The tele-symposium will feature talks by experts in projection mapping and by an interactive programmer of generative music applications.
\end{abstract}

Digital Art. Lighting. Color. Remote learning. Ubiquitous. Bangkok Design Week.

\section{INTRODUCTION}

Color Communication Design Laboratory in Japan, and Assumption University in Thailand co-hosted a tele-symposium and digital art workshops from December 2020 to March 2021. As an empirical case study, this paper describes these workshop and symposium remotely conducted. The purpose of this paper is twofold: firstly, to define the elements that are necessary for the future of international digital art workshops and symposia; secondly, to identify the advantages and problems and to apply them to future organisations. In particular, this presentation will focus on what communication platforms were used and how the themes were decided upon to create the works. Works resulting from these will be presented.

An essential aspect of this symposium and workshop was that the motivation to have a common theme as well as an easy-going collaborative project experience and also to show the results to the public has the effect of giving students the vision to present what they have learned in these remote classes to the world.

The Covid-19 pandemic, which has radically changed the world beyond 2020, has had a major impact on our workshops and symposia: from 2019 onwards, Assumption University, Montfort del
Rosario Since 2019, the School of Architecture and Design at Assumption University, Montfort del Rosario, outside Bangkok, Thailand, and the School of Design at Shibaura Institute of Technology, Tokyo, Japan, have co-organised two previous workshops and international symposiums on digital artmaking. The first took place in March 2019 at the Shibaura campus of the Shibaura Institute of Technology in Tokyo and the second in November 2019 at the Lido Connect, a now-former theatre in Bangkok's city centre at Siam Square. Each workshop has had a common theme and a total of around 30-40 students have worked in 6 teams to create a piece of work.

The third workshop in 2020 was originally supposed to be held locally, either in Japan or in Thailand. However, due to the effects of the Covid19 disaster, it was decided that the digital art workshop and the international symposium would be held entirely remotely.

The overall theme of the third workshop was "Ubiquitous Lighting". The word ubiquitous [ubi-quitous] is of Latin origin and means "omnipresent" (Hornby et al. 2013). The first theme was "Colorful Playground for Children" (Hidaka 2019) and the second theme was "Illuminate" (Assumption University 2019). 
The workshop was held between 23 November 2020 until 22 February 2021. The tele-symposium took place on 1 March 2021 to present and discuss the work together.

We were accepted to use a space to exhibit the actual artworks at Bangkok Design Week 2021 (Bangkok Design Week 2020) and the goal was to place an installation in this event.

Carnegie (1835-1919), an American-Scottish industrialist and philanthropist, spoke of the importance of teamwork and shared vision:

\begin{abstract}
Teamwork is the ability to work together toward a common vision. The ability to direct individual accomplishments toward organizational objectives. It is the fuel that allows common people to attain uncommon results.
\end{abstract}

\section{--Andrew Carnegie}

As Carnegie indicated, it is not possible to create art simultaneously and remotely without sharing a common vision.

\section{COURSE DESCRIPTION}

The workshop lectures started at 11am (9am in Bangkok) on 23 November 2020. There is a twohour time difference between Bangkok and Tokyo. The workshop was held every Monday morning and afternoon. The following is an outline of the class:

\begin{abstract}
This course is a seminar/workshop on interaction design, which is the study of systems and devices that a user can interact. The practice typically centres on embedding information/digital technology into various outputs in the physical world. The course also discusses about human behaviours, social interactions, and the impact of digital media on built environments. Students will be asked to develop their investigations and interests as well as a space for exploration, experimentation, and implementation. (AR 5415 Interactive Features Design, Assumption University)
\end{abstract}

The workshop aimed to design an interactive space and lighting. The Thai teachers were Joe Nattapol Suphawong, an interactive designer and photographer, and Daruswat Wattanarojjanananikorn, also an interactive designer. Apinantaya Bua-iem, Head of the Department, also participated as a supervisor. The Japanese team consisted of Kyoko Hidaka, a color theorist, and Takumi Miyoshi, a network specialist.

\subsection{Communication platforms}

The following platforms were used to communicate remotely for this workshop Microsoft Teams was used as the overall communication tool. It was the platform we needed for video streaming of classes, submitting reports and communicating with groups of students.

On the Japanese side, Slack was also used to provide a platform for collaborative work. Initially, face-to-face classes were possible in Thailand, and there were around 20 students in a classroom at Assumption University. At the same time, students who had not yet entered Thailand participated in the hybrid style, using Microsoft Teams from Vietnam, Taiwan and China. Shibaura Institute of Technology in Japan also participated with 5 undergraduate students and 2 technical assistants from remote locations via Microsoft Teams. In other words, nearly 30 people, including teachers, participated in this workshop every week.

However, since the end of December, a state of emergency has been declared due to the Covid-19 outbreak, and all Thai universities are now teaching remotely. The students who participated in the workshop were from various countries, including Thailand and Japan, Taiwan, China, Vietnam and Cambodia.

The international symposium, which was also the final presentation, was delivered by Shibaura Institute of Technology using Zoom (Figure 1).

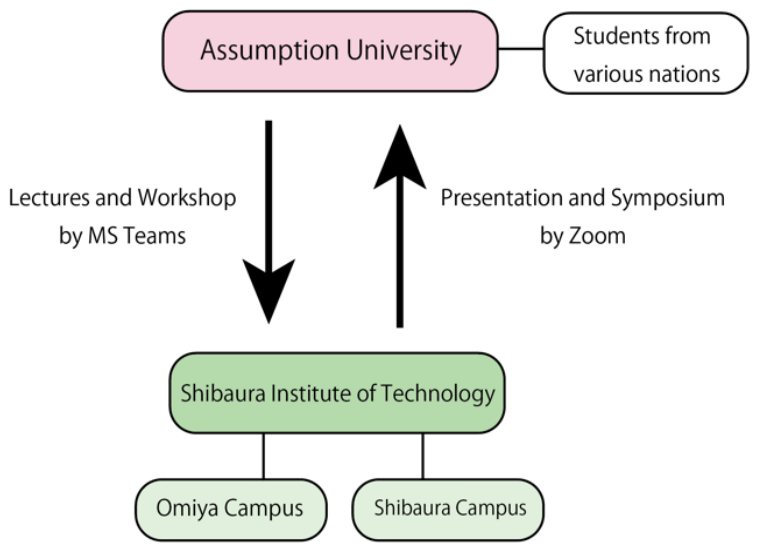

Figure 1: System configuration

\subsection{Group Themes}

At Assumption University, we were first divided into four groups. Apart from the overall theme of "Ubiquitous Lighting", each group was given a predefined theme by their instructors, which positioned them in the following technological directions:

- Group I: Hello World

- Group II: Information Aesthetics

- Group III: Telepresence

- Group IV: Networked/Cultural Light 
The students were not necessarily bound to any of these themes, but it was useful for each group to have its own theme in order to avoid a similar orientation.

The Bangkok Design Week was originally scheduled to start at the end of January. However, due to the sudden finding of an outbreak of Covid19 in the suburbs of Bangkok at the end of December, university classes were suddenly changed to remote learning only, and the Design Week was postponed until May.

\section{WORKSHOP}

\subsection{First assignment}

The first homework assignment for all the students was to define digital art in their way and to write a report on three case studies of digital artworks of interest from around the world, which the students had to research on the Internet.

For example, one student presented "Les Archives du Cœur (Archives of heartbeats)" by Christian Boltanski, a work that uses spatial lighting to represent the human heartbeat. Another work was "Deathclock for participation" by Tatsuo Miyajima, in which the audience takes a selfie and enters a certain date and time as the time of death, which starts a countdown. Other students described 'Technological Nature' by media artist Daria
Jelonek, which uses mirrors and telephones to represent the glowing phenomena of nature.

Through this assignment, the students gained a deeper understanding of the representation of interactive and digital art in different countries.

\subsection{Second assignment}

The next homework assignment was for each group to give a Powerpoint presentation on the design and concept of the piece they intended to create.

\section{WORKSHOP PRESENTATION}

The tele-symposium consisted of a live streamed presentation of student work in the first half and listening to guest speakers' presentations and discussions in the second half (Figure 2).

\subsection{Student presentation by Assumption University}

\subsubsection{Group 1}

The group produced a sphere, entitled 'Hello World', which lights up when people wave their hands. When the large sphere lights up, it sends a signal to the small sphere, which also flashes. The idea is to bring a connection between people who have to be separated by social distance (Figure 3 ).
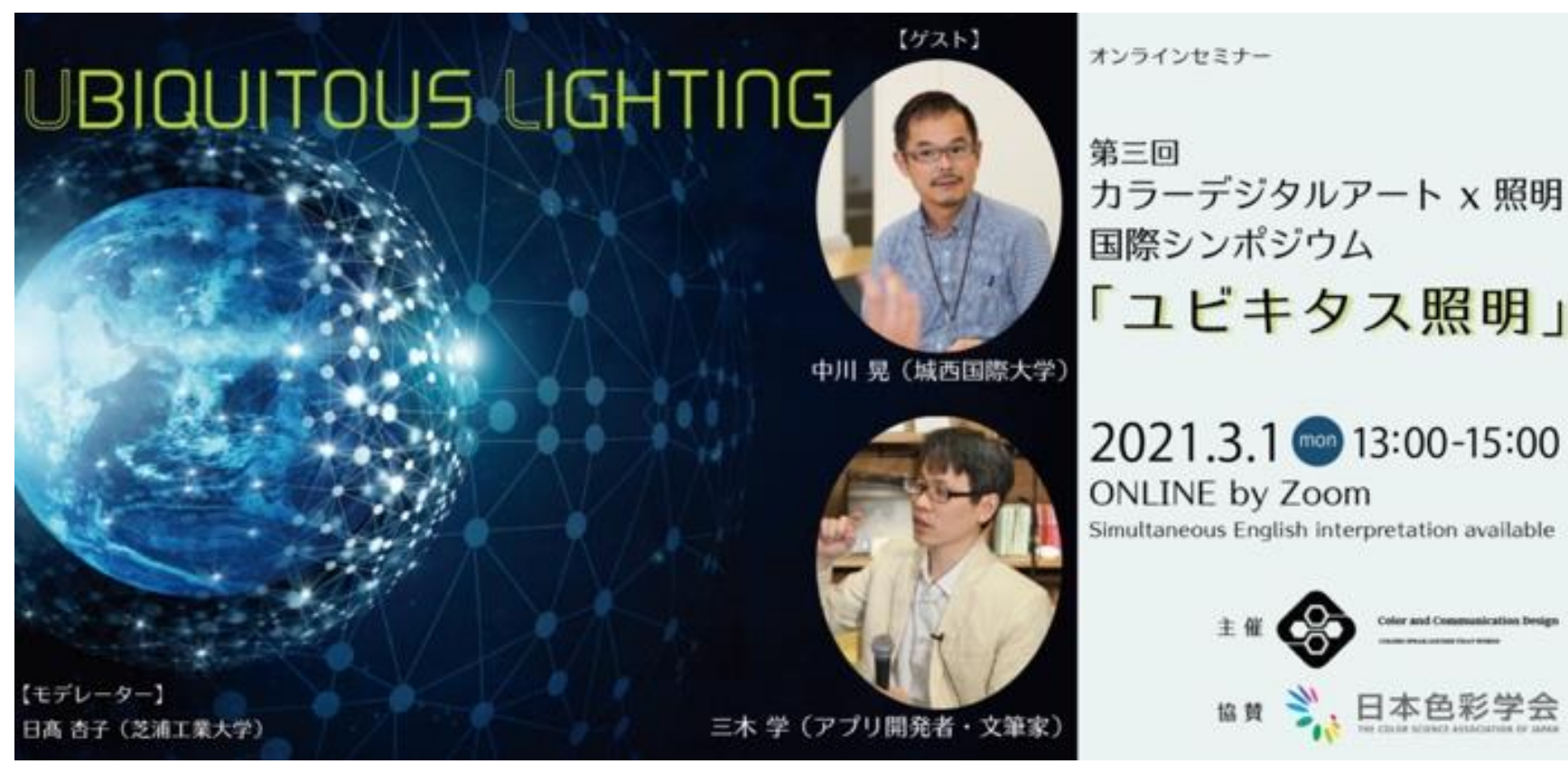

Figure 2: Screenshot of web advertising banner for the Third International Symposium. 


\subsubsection{Group 2}

Group 2's work was based on the concept of 'Whatever Light', in which light sculptures respond to the light from a person's smartphone, playing with the interaction of the patterns created by the light and shadow created by the three sculptures (Figure 4).

\subsubsection{Group 3}

Group 3 created 'The Magic Donation Box', a box that, when filled with coins, illuminates with lights representing positive words. The intention is to help people who are suffering from financial and emotional difficulties due to the pandemic (Figure 5).

\subsubsection{Group 4}

Group 4 members produced remotely, most of whom live outside of Thailand. Based on the theme of 'Cultural Light', the lights in the terminals at the university in Bangkok are illuminated by the movements of the people on the Zoom screen (Figure 6).

\subsection{Student presentation by Shibaura Institute of Technology (SIT)}

\subsubsection{Group SIT Omiya Campus}

The student work by the School of System Engineering and Science of Shibaura Institute of Technology used two terminals. The first terminal is an Arduino with a Bluetooth module and 4 LEDs, this terminal can be connected to a mobile battery for easy transport. The other terminal is a windows pc with Bluetooth This terminal can be connected to a mobile battery for easy transportation. At first, the Arduino terminal ' $A$ ' does not light up when the two terminals are in the distance where Bluetooth communication is not possible. Next, when ' $A$ ' approaches ' $\mathrm{B}$ ' and enters the range where Bluetooth can be connected, he receives a packet from the PC and the LED lights up. When the Bluetooth connection is lost, the light goes off.

For this work, they used Bluetooth network. Bluetooth is suitable for communication in a small area because it consumes less power and is not affected by the environment (Figure 7).

\subsubsection{Group SIT Shibaura Campus}

The team of the College of Engineering and Design of Shibaura Institute of Technology has developed a lighting design based on the motif of a planetary system, using the keyword ubiquitous, which means widely spread and everywhere. The rainbow-colored ring, which incorporates the central planet, represents the orbit of the planetary system. The orbits of the planets always revolve regularly around the planets and exist primarily with the planets. They associated this with the word ubiquitous and decided to represent the planets. The most ingenious part of the project was to use an Arduino to control the transition of the color of the LED tape so that it changes in a gradation. To make the LED tape light up at the same time, we made a file and rewrote the code accordingly. We used two devices for this work: the first one is an Arduino with a Bluetooth module and 4 LEDs, connected to a mobile battery and easily portable (Figure 8).

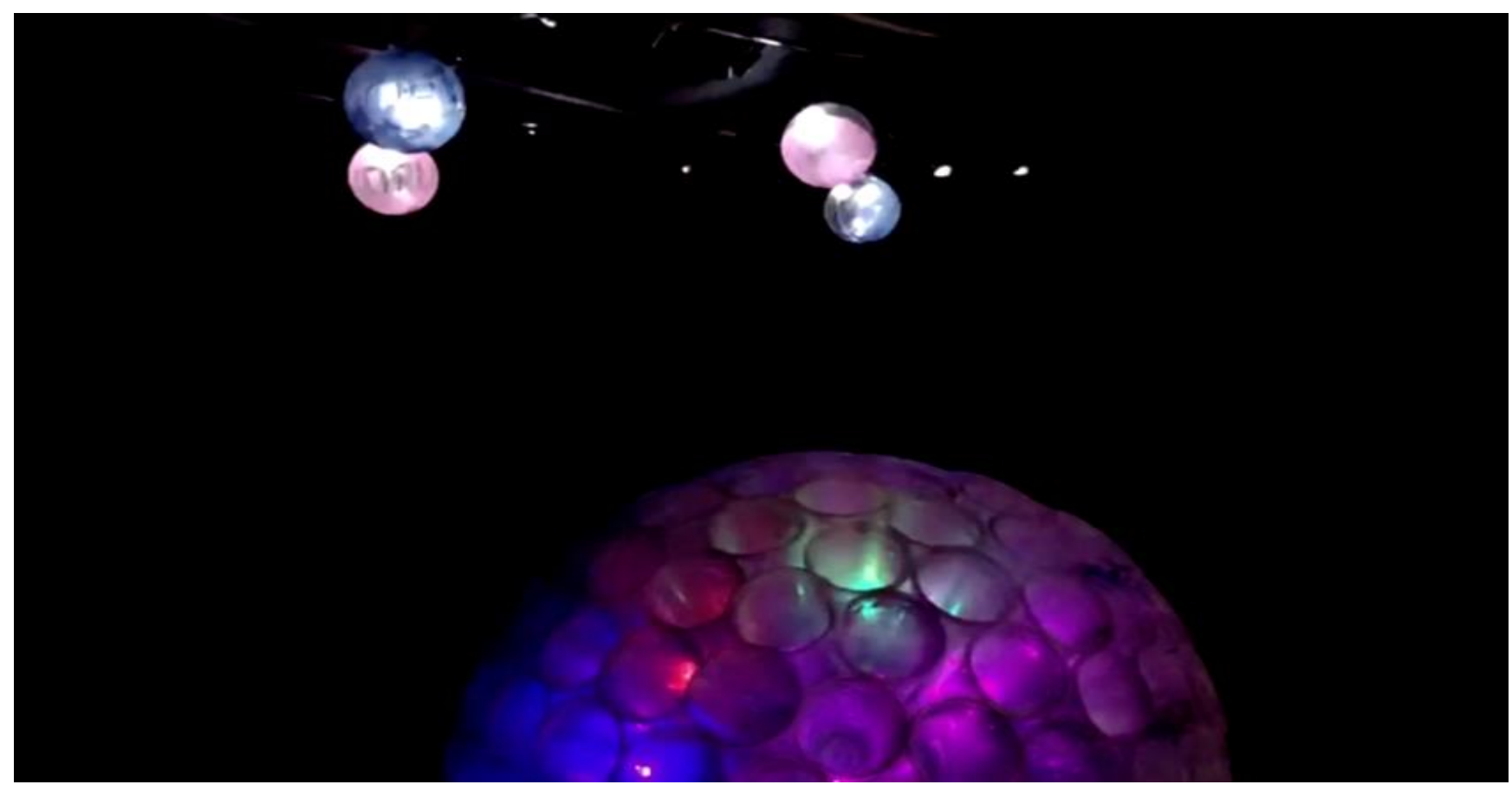

Figure 3: Hello World (Group 1, Assumption University) 


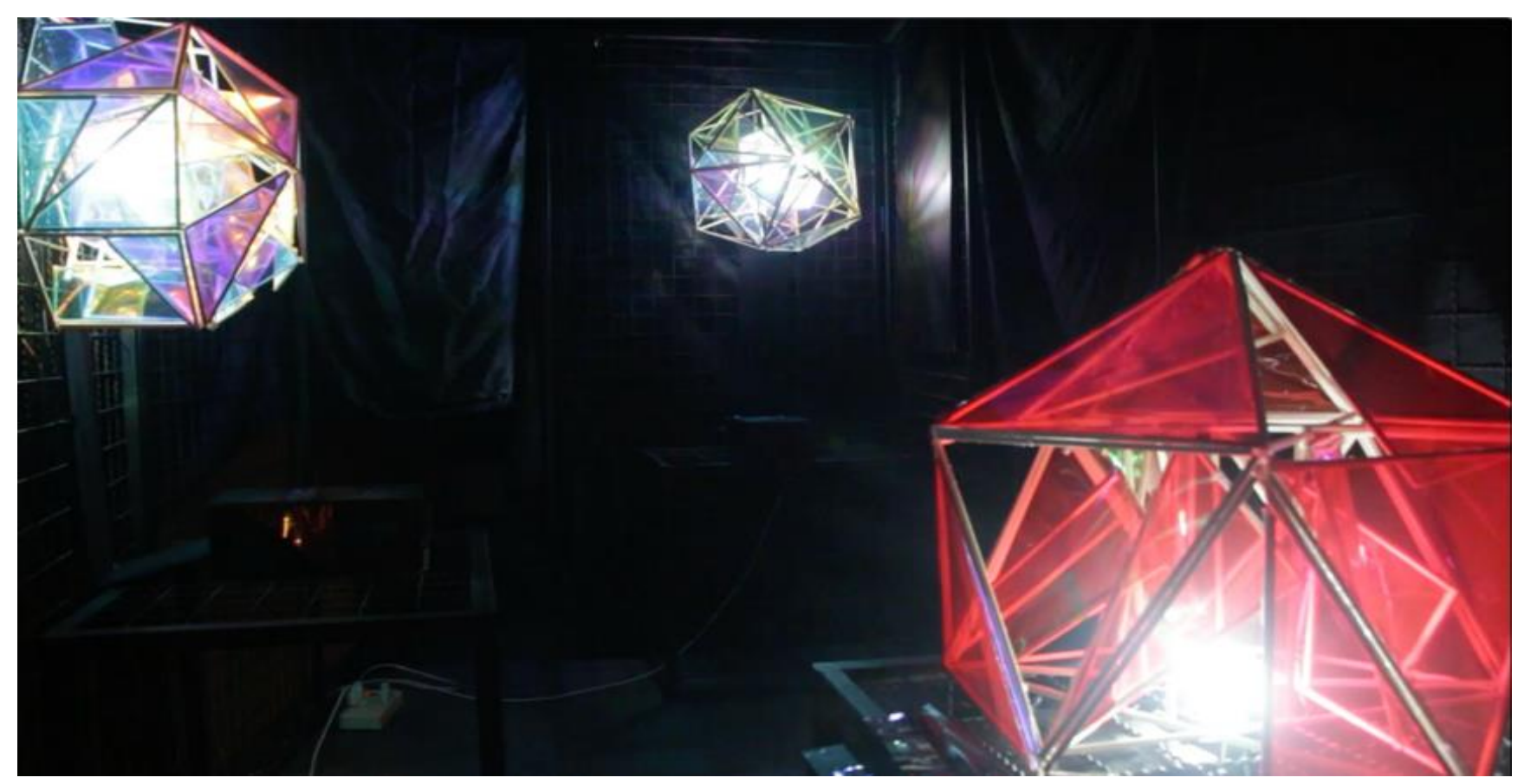

Figure 4: Whatever Light (Group 2, Assumption University)



Figure 5: Magic Donation Box (Group 3, Assumption University) 


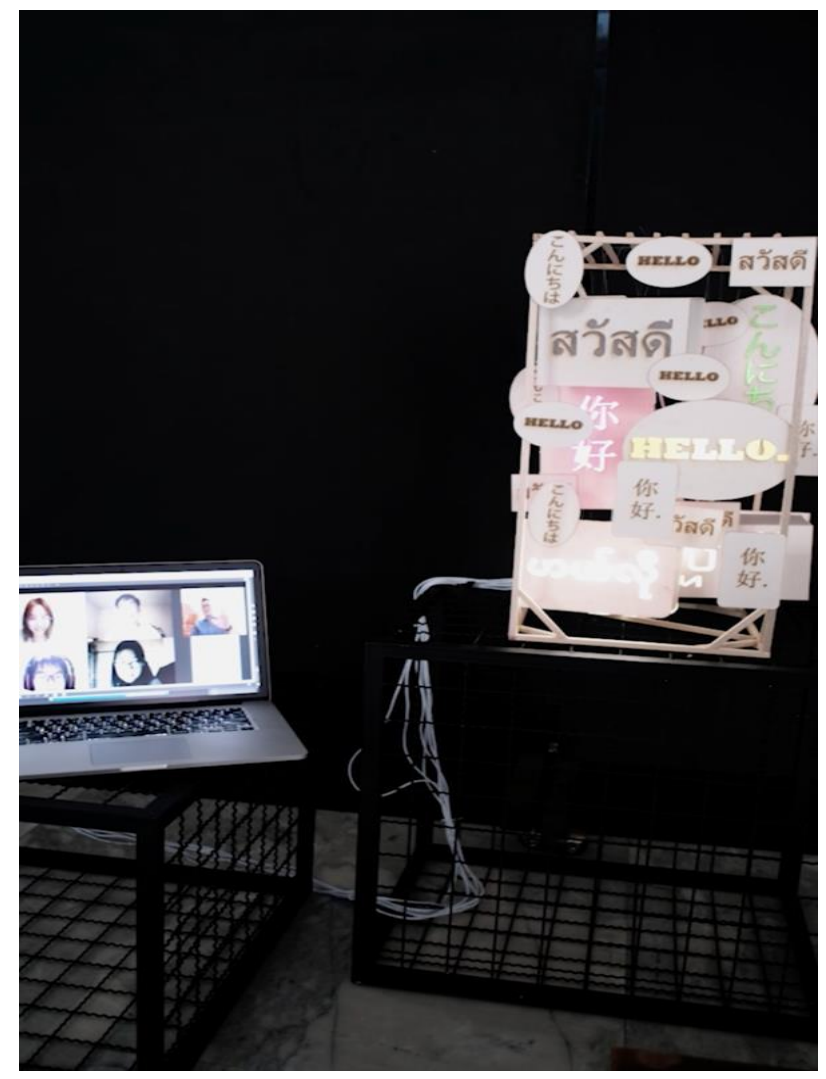

Figure 6: Cultural Light (Group 4, Assumption University)

\section{GUEST PRESENTATIONS}

During the symposium, two guests were invited for presentations and discussions. The Moderator was Prof. Kyoko Hidaka. The following are the guests.

- Akira Nakagawa (Josai International University)

- Manabu Miki (art critic, apps programmer)

\subsection{Akira Nakagawa}

One of the symposium's guest speakers, Nakagawa, is a supervisor at Josai International University, where he won the prize for excellence in the 2020 Tokyo Projection-Mapping Awards. He spoke about his experience of remotely producing digital art. He worked with students to create a projection mapping project on the theme of "SHAPE of SOUNDs". According to Akira Nakagawa, we spent a lot of time thoroughly examining "understandability" and explaining how they would perceive it.

Nakagawa specialises in stage and television art, advertising and media studies. He started his career working for a Japanese national television station, then moved to a company managing Disneyland, and is now a university teacher.

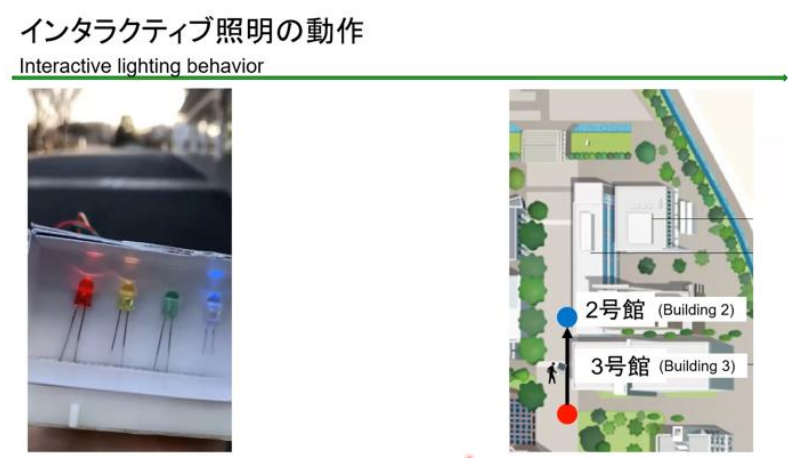

Figure 7: Location-based Ubiquitous Lighting using Bluetooth (Shibaura Institute of Technology, Omiya Campus)

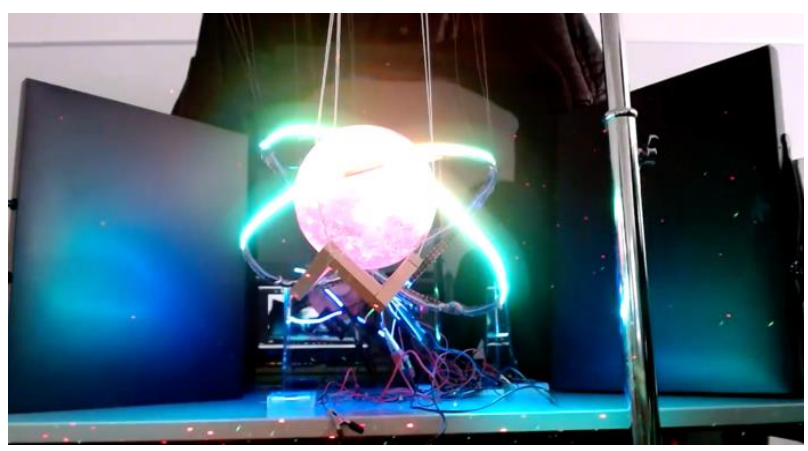

Figure 8: Ubiquitous Planet (Shibaura Institute of Technology, Shibaura Campus)

The competition was held in Odaiba area in Tokyo, and in 2020 roughly 30 schools from the UK, Canada and Japan entered. Projection mapping images were projected onto the walls of a building called Big Sight. It was the first time for Nakagawa and his team to participate in the event, and the main topic of discussion was how they managed to create a highly acclaimed piece of work with no know-how and only about 20 students in their year group.

In any production, the Covid-19 pandemic is a major distinctive factor in 2020. When we entered the competition, the most important thing for us was to set up the concept. The theme of this year's competition was "connect with". Nakagawa and his team devised a concept in line with this theme: 'After much discussion, they decided on the concept 'SHAPE of SOUNDs'. The concept was based on the idea that it would be interesting to make visible the various sounds in our daily lives that we are not usually aware of.

\subsection{Manabu Miki}

Miki gave a presentation with the following title: "New Phantasmagoria and Synesthesia Art". Phantasmagoria is a spatial projection technology show that originated in $18^{\text {th }}$-century France and became popular in $19^{\text {th }}$-century England. As a form of horror theatre or haunted house, it is a medium in which images of skeletons, demons and ghosts 
are projected onto walls, smoke and translucent screens using magic lanterns (the ancestor of projectors). The images of ghosts were projected from behind a partition or something similar. It is said to be the forerunner of the film, and the projector evolved from here.

In the second half of the talk, Miki introduced his work on the sonification of images and applications. By programming colors with generative scales, Miki is trying to create sounds converted from human life scenes. (DOZAN11, et.al. 2019)

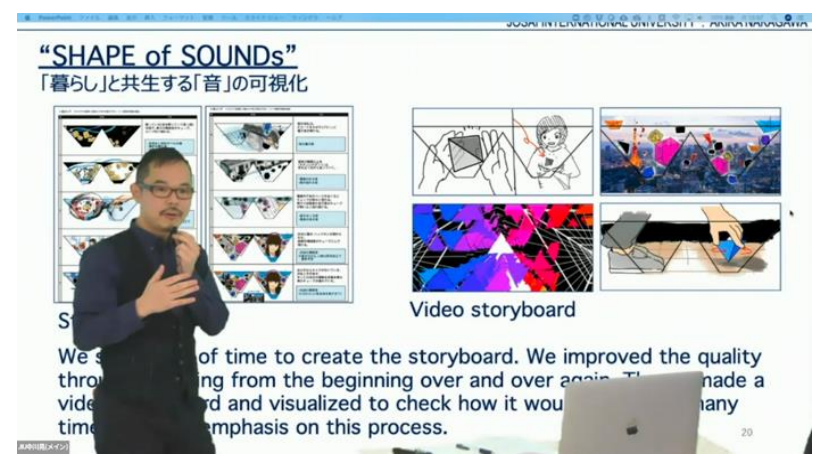

Figure 9: Screenshot of Nakagawa's presentation

\subsection{Guest discussion}

The questions for the symposium discussion were as follows:

(a) What is the most enjoyable experience you have ever had?

(b) What are the 'experiences' and interactions when working remotely and collaboratively?

(c) How do you see future events being organised at the Pandemic?

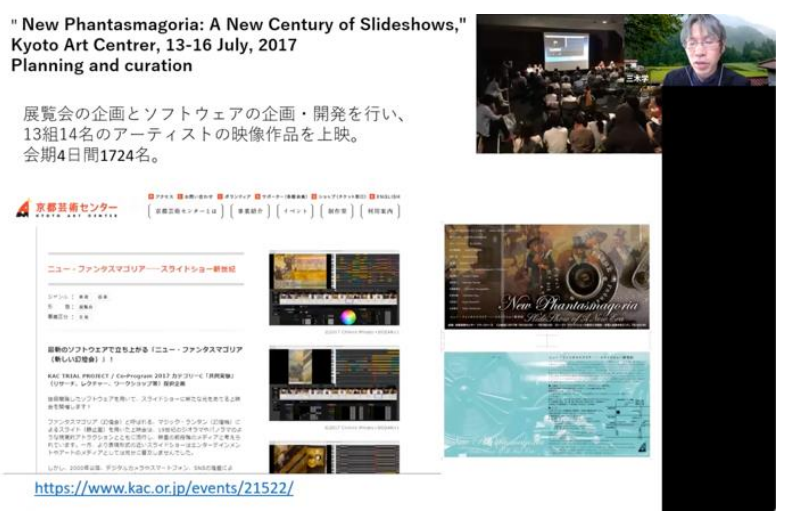

Figure 10: Screenshot of Miki's presentation

The willingness to share the goals Nakagawa talked about in his presentation and to work together as one to achieve the same goal is the most key to remote work. It seems to be possible to create without being physically in the same place. Paradoxically, if you are with a stranger on a train, for example, and you don't share any ideas, nothing will come of it. We are teleworking, but we have participants from different places like Thailand, Vietnam and Taiwan. Besides, ideas are shared through interpreters and subtitles. More than the technology of interactive art and media art, it is important to share a clear metaphysical concept.

The guests were asked to talk freely about their predictions for the future of digital art production, and what Nakagawa and Miki imagine the future to be like.

Miki explained that the history of projection has always been marked by the World Exposition, especially which was held in Osaka in 1970, and will be held again in 2025. In 1970, the Osaka Expo attracted up to 800,000 people a day to the same place. He forecasted that the Expo of the 21st century will create a situation where people can share experiences to some extent without having to be physically there. Participation used to be a matter of going to one place altogether. In the future, however, events will become ubiquitous, meaning that people will be able to experience them anywhere.

Nakagawa explained that there are two aspects of the future that he sees: one is an environmental future, and the other is a changing human future. Technology and environmental changes are evident in the results of the year 2020, with the $5 \mathrm{G}$ communication environment being a typical example of the situation. The world tour of South Korean popular singers BTS was cancelled, but thanks to communication technology, it was able to reach more people than it should have. The production of the world tour was so good that society thought it was better than a real concert.

Using epic games' Fortnite as a platform, the live broadcast allows guests to create avatars and participate as a different person. The satisfaction rate of physical live games is not that high, but the satisfaction rate of virtual live games is over $95 \%$.

There is a situation where the virtual live is more satisfying. Therefore, we believe that the future will be a hybrid of both. If the sound quality of video distribution is improved and the problem of reality is solved, both real and virtual events will be possible.

The environment for events will certainly change over the next 10 to 20 years. Generation $Y$ is the generation of digital natives, while Generation $Z$ is the generation of empathy, where live streaming on TikTok is still the norm.

When Nakagawa was young, in his 40s, he was given the idea that TV was a mass medium and that what everyone else thought was good was good. Nowadays, however, there is a culture of 
accepting what you think is good for you. We live in a society where anyone can deliver a live performance, even if there are only five people in the audience.

Many generations have believed that a live performance had to be a finished work, but now the younger generations do not care if it's not a completed work, and they can enjoy it even if it's just a businessman broadcasting something from the comfort of his room during the day. For the older generation, it is difficult to understand, but for the younger generation, it is not so strange. The development of technology is changing the way we perform and the environment. There will be a time when big events and small events will go hand in hand, both on the internet and in real life. The possibilities of digital art are huge, from large-scale projection mapping on buildings to share your memories, photos and music with the world on your phone.

\section{CONCLUSION}

In conclusion, it has been possible to see a future in which the sharing of visions and the hybridisation of the virtual and the real, even remotely, will surely continue to grow.

By presenting the work not just during the workshop as a production, but also at the symposium, we could hear the perspective of a third party. We were able to revisit the results of the workshop from the perspective of projection mapping, which is the visualisation of sound, and applications and expositions, which are the sonification of images.

The environment in which individuals can easily broadcast their daily lives and emotions through the internet, for example by turning their memories into sounds, is considered to be more advanced than before the pandemic.

Furthermore, it is becoming more and more widespread to be able to give lectures and watch artworks virtually, from the comfort of one's own home or laboratory, without having to rent some venue, university auditorium or theatre. While there is certainly a high value in physically visiting a site to experience it, we can observe an increase in the range of experiences and education that individuals can enjoy at home.

The technological limit in which artworks can be broadcasted only through complicated skill should be removed, and that an information about digital art that can be easily disseminated worldwide and experienced by individuals will advance.

\section{REFERENCES}

Assumption University. (2019) The $2^{\text {nd }}$ Digital Art $x$ Lighting Workshop \& Symposium. Conference website:

http://www.arch.au.edu/illuminateworkshop2019 (retrieved 12 March 2021).

Bangkok Design Week (2021) Conference website: https://www.bangkokdesignweek.com/ (retrieved 12 March 2021).

Bullivant, L. (2005) 4dspace: Interactive Architecture (Architectural Design). Wiley, Hoboken, New Jersey.

DOZAN11 (2019). DIVA Co and Visionarist Co., Ltd. Online directory: https://mupic.jp (retrieved 16 March 2021).

Hidaka, K. (2020) Symposium Dialog. Color and Communication Design 1, pp.1-13.

Hidaka, K. (2019) Fusion of Art and Technology: The First Color Digital Art x Lighting Symposium and Workshop. 2nd International Conference, Digital Culture \& AudioVisual Challenges, Interdisciplinary Creativity in Arts and Technology (DCAC 2019), Corfu, Greece, 10-11 May 2019. Ionian Academy of the Ionian University, Corfu.

Hornby, A. et al. (2013) Oxford Advanced Learner's Dictionary. Oxford University Press, Oxford.

Paul, C. (2015) Digital Art (World of Art Book). Thames and Hudson Ltd, New York. 